\title{
Data-driven Green's function retrieval and imaging with multidimensional deconvolution: numerical examples for reflection data with internal multiples
}

Filippo Broggini* and Roel Snieder, Center for Wave Phenomena - Colorado School of Mines;

Kees Wapenaar, Delft University of Technology

\section{SUMMARY}

Standard imaging techniques rely on the single scattering assumption. This requires that the recorded data do not include internal multiples, i.e. waves bouncing multiple times between layers before reaching the receivers at the acquisition surface. When multiple reflections are present in the data, standard imaging algorithms incorrectly image them as ghost reflectors. These artifacts can mislead the interpreters in locating potential hydrocarbon reservoirs. Recently, we introduced a new approach for retrieving the Greens function recorded at the acquisition surface due to a virtual source located at depth. Additionally, our approach allows us to decompose the Green's function in its downgoing and upgoing components. These wave fields are then used to create a ghostfree image of the medium with either crosscorrelation or multidimensional deconvolution, presenting an advantage over standard prestack migration. We illustrate the new method with a numerical example based on a modification of the Amoco model.

\section{INTRODUCTION}

One of the main goals of exploration geophysics is to retrieve the location and amplitude of the discontinuities between layers with different properties inside the subsurface from reflection data acquired on the surface of the earth. This is a challenging research task because an accurate image of the structures inside the earth is needed to locate energy sources, such as hydrocarbon reservoirs.

Standard imaging methods, such as one-way prestack migration (Claerbout, 1985), usually suffer from artifacts (ghost images) due to the presence of multiple reflections in the recorded data. These multiple reflections are often caused by high impedance layers, such as salt bodies, and hence they can severely affect the final images. One-way imaging schemes cannot differentiate between primaries and multiples; hence they erroneously interpret the multiply reflected waves as primary reflections due to deeper layers.

We have recently introduced a new method that allows us to reconstruct the total wave field originating from a point in the subsurface to receivers located at the surface (Wapenaar et al., 2012, 2013). The Green's function, defined as the response recorded at the acquisition surface due to a source located in the interior of the subsurface, see Figure 1a, is a combination of the downgoing and upgoing wave fields needed to reconstruct an image of the discontinuities inside the earth. These downgoing and upgoing wave fields are illustrated in Figure 1b, where we have applied source-receiver reciprocity. The new method consists of an iterative algorithm that transforms the reflection response (at the acquisition surface) of an arbitrary medium into the wave field generated by a virtual source inside the unknown medium. Apart from requiring the reflection data measured at the surface, the proposed method also requires an estimate of the traveltimes of the first arriving wave traveling from the virtual source location to receivers located at the acquisition surface. These traveltimes are

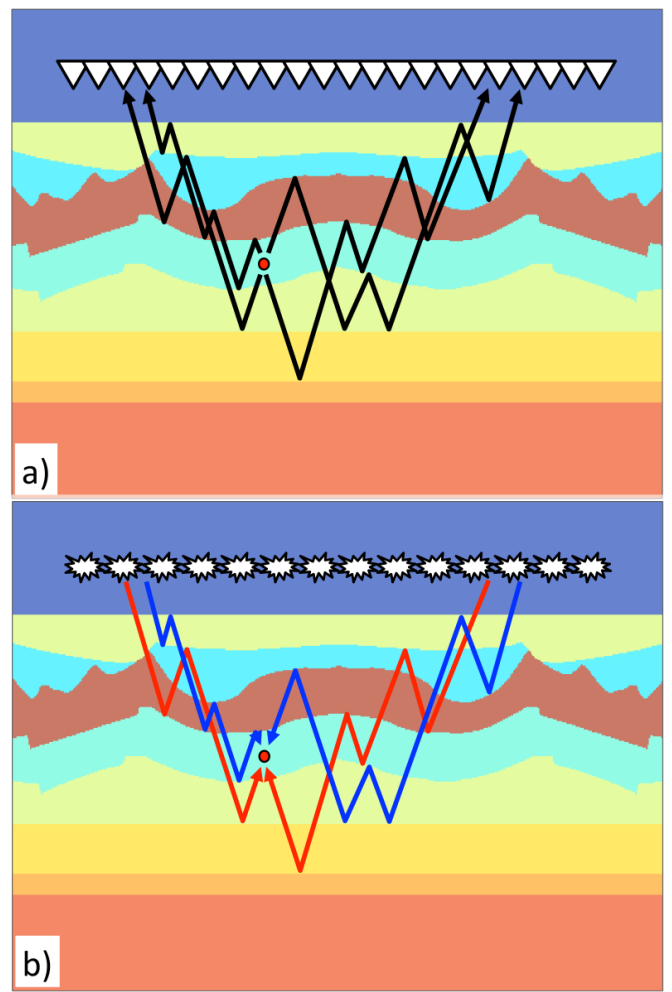

Figure 1: a) Total wave field from the imaging point located at $\mathbf{x}_{I}$ (indicated by the red dot) and recorded at the acquisition surface. b) Decomposed wave fields. The solid blue rays correspond to the downgoing component $G^{+}\left(\mathbf{x}_{I}, \mathbf{x}, t\right)$. The solid red rays correspond to the upgoing component $G^{-}\left(\mathbf{x}_{I}, \mathbf{x}, t\right)$.

a key element of the method because they specify the location of the virtual source in the subsurface. Consequently, the proposed method is not fully model-independent. However, we do not require any more knowledge of the medium parameters than standard primary imaging schemes. We emphasize that we use the internal multiples to construct images with more correct amplitudes, while other methods aim at removing the internal multiples before the imaging step (Berkhout and Verschuur, 1997; Weglein et al., 1997; Jakubowicz, 1998; ten Kroode, 2002). Moreover, our method is non-recursive, which means that it does not suffer from error propagation. Also we do not need to resolve the multiple problem in the overburden prior to imaging a specific target zone.

\section{CONFIGURATION}

We consider a model based on a modified version of the Amoco dataset, originally introduced by Etgen and Regone (1998), as shown in Figure 2. Since our method allows targeted imaging, we focus on the portion of the model enclosed by the dashed rectangle in Figure 2. The velocity and density profiles for this portion of the model are shown in Figures $3 \mathrm{a}$ and $3 \mathrm{~b}$. The black arrow in 


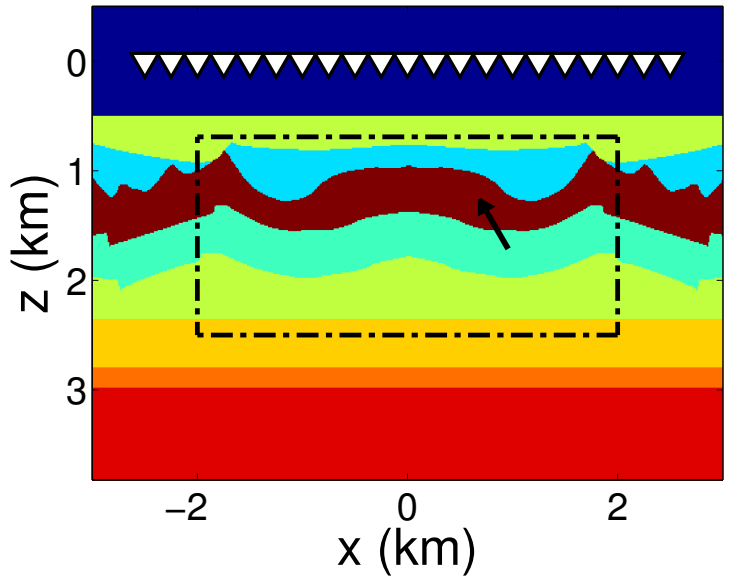

Figure 2: Velocity model. The white triangles denotes the receivers at $z=0$. The black arrow indicates a layer characterized by higher impedance with respect to the surrounding layers.

Figure 2 indicates a layer characterized by higher impedance with respect to the surrounding layers.

We computed 501 shots with sources located at $z=0 \mathrm{Km}$. The acquisition surface is transparent; hence no surface-related multiples are present. In the horizontal direction, the sources are located every $10 \mathrm{~m}$ between -2.5 and $2.5 \mathrm{Km}$. Alternatively, the required reflection responses are obtained from conventional seismic reflection data after removal of the multiples due to the free-surface and after deconvolution for the source wavelet (Verschuur et al., 1992; Amundsen, 2001; van Groenestijn and Verschuur, 2009).

We compute the direct arriving wave front, required by our method, using an eikonal solver. As in input for the solver, we use a smooth version of the velocity model; see Figure $3 \mathrm{c}$. Note that, using an eikonal solver, we ignore the influence of the density. Alternatively, the direct arriving wave front can be obtained by modeling in a macro model, from microseismic events (Artman et al., 2010), from borehole check shots, or directly from the data by the CFP method (Berkhout, 1997; Thorbecke, 1997; Haffinger and Verschuur, 2012) when the imaging point is located at an interface.

\section{IMAGING}

\section{Standard prestack imaging}

We start by creating a reference image using a standard imaging technique (Claerbout, 1985). Standard prestack imaging and many other seismic imaging algorithms rely on the single scattering assumption. This implies that the recorded wave fields do not include internal multiples (waves bouncing multiple times between reflectors before reaching the receivers). When unwanted multiple reflections are present in the data, the imaging algorithm incorrectly images them as ghost reflectors. This reference image is shown in Figure 6a, where we have used the same smooth model needed to compute the first arriving waves needed by the iterative scheme; see Figure 3c. The high-impedance layer, indicated by the black arrow in Figure 2, creates high-amplitude multiply-scattered waves. The yellow arrows in Figure 6a indicate some of the ghost reflectors present in this standard image. Additionally, the image is more noisy below the high-impedance

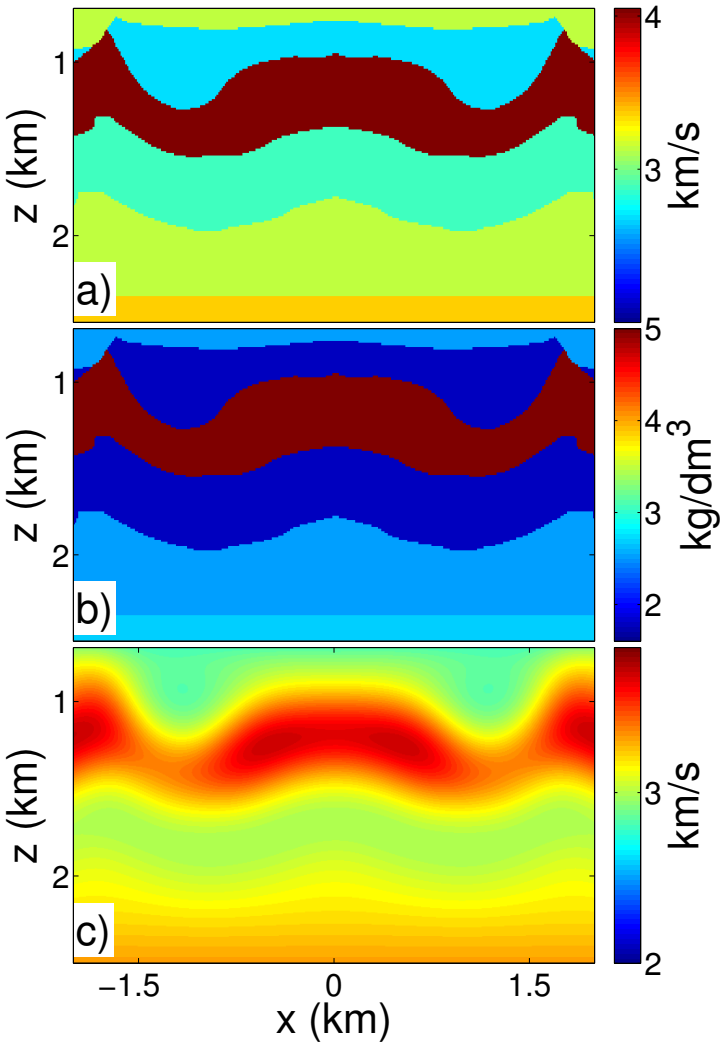

Figure 3: a) Velocity model. b) Density model. b) Smooth velocity model.

layer. The ghost reflectors and the noise mask the flat reflector at $z=2.3 \mathrm{~km}$, indicated by the blue arrow in Figures $6 \mathrm{~b}-\mathrm{c}$, and affect the lateral continuity of the deeper reflectors.

\section{Imaging with crosscorrelation}

If we retrieve the Green's function with our method and decompose it in its downgoing and upgoing components for imaging points $\mathbf{x}_{I}$ located on many depth levels $z=z_{i}$ (e.g. the horizontal lines composed of black dots in Figure 4), we are able to create a more accurate image of the medium. To perform this task, we compute the crosscorrelation function $C$

$$
C\left(\mathbf{x}_{I}, \mathbf{x}_{I}, t\right)=\int_{-\infty}^{\infty}\left[G^{-}\left(\mathbf{x}_{I}, \mathbf{x}, t\right) * G^{+}\left(\mathbf{x}_{I}, \mathbf{x},-t\right)\right]_{z=0} \mathrm{~d} x
$$

at every imaging point depth and evaluate the result at $t=0$. This new image is shown in Figure 6b. As in the standard prestack image, the actual reflectors have been reconstructed at the correct spatial location, but now the image is free of internal multiple ghosts. The image is now less noisy and the flat reflector at $z=2.3$ $\mathrm{km}$ is more visible. This is an improvement over the previous image, but the retrieved amplitudes of the deeper targets are not consistent with the true reflection coefficients. This is due to fact that we have a limited source aperture and do not take into account the multi-dimensional nature of the seismic wave field.

\section{Imaging with multidimensional deconvolution}

Now, we show that multidimensional deconvolution allows us to create an image with more accurate amplitudes. As in imaging with crosscorrelation, we consider a constant depth level $z=z_{i}$. 


\section{Green's function retrieval and imaging with MDD}

The Green's functions at this constant depth level are related by

$$
G^{-}\left(\mathbf{x}_{r}, \mathbf{x}_{s}, t\right)=\int_{-\infty}^{\infty}\left[R\left(\mathbf{x}_{r}, \mathbf{x}, t\right) * G^{+}\left(\mathbf{x}, \mathbf{x}_{s}, t\right)\right]_{z=z_{i}} \mathrm{~d} x,
$$

where $R\left(\mathbf{x}_{r}, \mathbf{x}, t\right)$ is the reflection response to downgoing waves at $z=z_{i}$ of the truncated medium below $z=z_{i}, \mathbf{x}_{s}$ is at $z=0$, and $\mathbf{x}_{r}$ is at $z=z_{i}$. The truncated medium is equal to the true medium below the depth level $z=z_{i}$ and is homogeneous above $z=z_{i}$. Now, the reflectivity $R\left(\mathbf{x}_{r}, \mathbf{x}, t\right)$ can be resolved from equation (2) by multidimensional deconvolution (MDD) (Wapenaar et al., 2011). To achieve this result, we first correlate both sides of equation (2) with the downgoing Green's function and integrate over source locations over the acquisition surface:

$$
\begin{gathered}
C\left(\mathbf{x}_{r}, \mathbf{x}^{\prime}, t\right)=\int_{-\infty}^{\infty}\left[R\left(\mathbf{x}_{r}, \mathbf{x}, t\right) * \Gamma\left(\mathbf{x}, \mathbf{x}^{\prime}, t\right)\right]_{z=z_{i}} \mathrm{~d} x, \\
\text { for } \mathbf{x}_{r} \text { and } \mathbf{x}^{\prime} \text { at } z=z_{i},
\end{gathered}
$$

where $C$ is the crosscorrelation function (as in the previous section, but with not-coinciding coordinates)

$$
C\left(\mathbf{x}_{r}, \mathbf{x}^{\prime}, t\right)=\int_{-\infty}^{\infty}\left[G^{-}\left(\mathbf{x}_{r}, \mathbf{x}_{s}, t\right) * G^{+}\left(\mathbf{x}^{\prime}, \mathbf{x}_{s},-t\right)\right]_{z_{s}=0} \mathrm{~d} x_{s},
$$

and $\Gamma$ is the point-spread function (van der Neut et al., 2011)

$$
\Gamma\left(\mathbf{x}, \mathbf{x}^{\prime}, t\right)=\int_{-\infty}^{\infty}\left[G^{+}\left(\mathbf{x}, \mathbf{x}_{s}, t\right) * G^{+}\left(\mathbf{x}^{\prime}, \mathbf{x}_{s},-t\right)\right]_{z_{s}=0} \mathrm{~d} x_{s} .
$$

We solve for $R\left(\mathbf{x}_{r}, \mathbf{x}, t\right)$ at different depth levels $z=z_{i}$ and many imaging points, as shown by the horizontal lines composed of black dots in Figure 4, and evaluate the result at $t=0$ and $x_{r}=x$. Figure $6 \mathrm{c}$ shows the final result of the imaging process. As in the previous image, the reflectors have been imaged at the correct spatial locations, but now the relative amplitudes between the different reflectors are better retrieved. Additionally, note how the lateral continuity of the deeper reflectors is well preserved in comparison with the prestack image in Figure 6a. The comparison between the amplitudes of the three images is shown in Figure 5, where we compare the reflectivity at $x=200 \mathrm{~m}$ retrieved by standard prestack migration, crosscorrelation, and multidimensional deconvolution. Multidimensional deconvolution acknowledges the multi-dimensional nature of the seismic wave field, hence the internal multiples contribute to the restoration of the amplitudes of the reflectors.

\section{CONCLUSIONS}

We showed that our recently introduced method for data-driven Green's function retrieval (Wapenaar et al., 2012, 2013) allows us to construct an image that is not affected by ghost images of the reflectors. The retrieved downgoing and upgoing components of the Green's functions are the key components needed to obtain this result. These wave fields are then used to create different images of the medium with crosscorrelation and multidimensional deconvolution. The images created with crosscorrelation and multidimensional deconvolution show an improvement over standard imaging. Additionally, multidimensional deconvolution yields an image whose amplitudes agree with the correct reflection responses. This is due to the deconvolution process that correctly handles the internal multiples and retrieves the correct amplitudes of the primary reflections. We emphasize that, for the crosscorrelation and multidimensional deconvolution results, the imaging process does

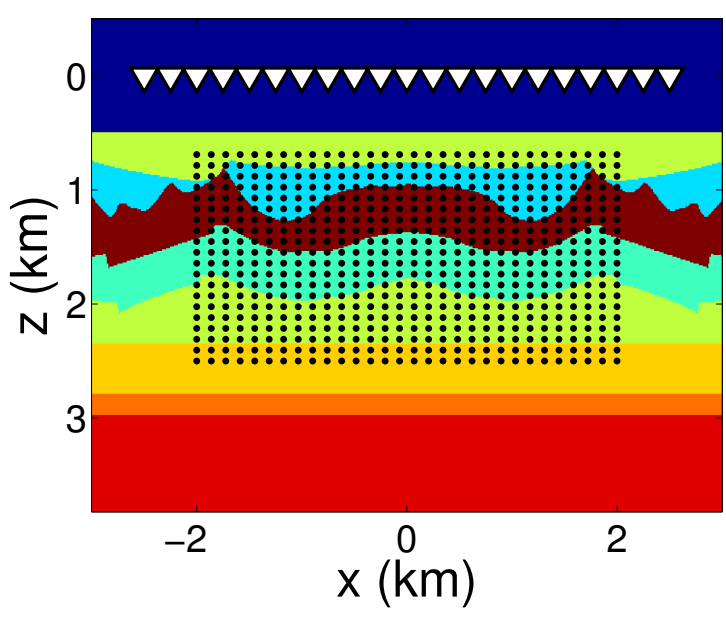

Figure 4: The black dots correspond to various imaging points $\mathbf{x}_{I}$. Imaging points located on a constant depth level $z=z_{i}$ are used to resolve $\left[R\left(\mathbf{x}_{r}, \mathbf{x}, t\right)\right]_{z=z_{i}}$.

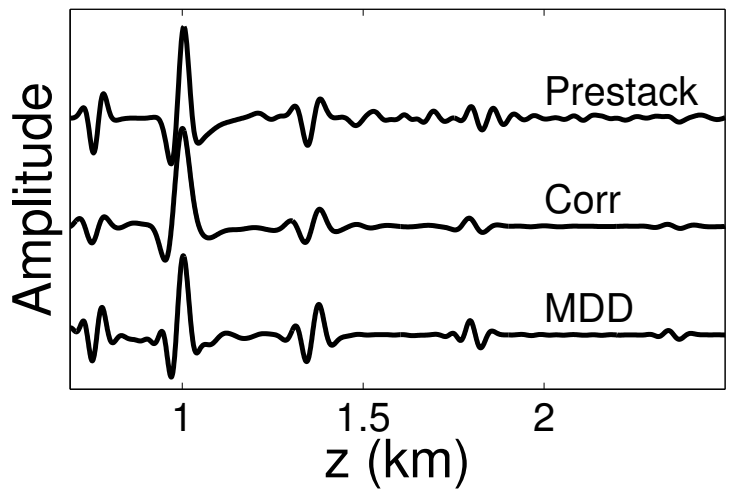

Figure 5: Comparison of the reflectivity at $x=200 \mathrm{~m}$ retrieved by standard prestack migration, crosscorrelation, and multidimensional deconvolution (from top to bottom).

not require any more knowledge of the medium parameters than standard primary imaging schemes (which use a macro model). Our method can be helpful in situations where waves have traversed a strongly inhomogeneous and scattering overburden, e.g., subsalt (Sava and Biondi, 2004) and near-surface imaging (Keho and Kelamis, 2012).

\section{ACKNOWLEDGMENTS}

This work was supported by the sponsors of the Consortium Project on Seismic Inverse Methods for Complex Structures at the Center for Wave Phenomena and the Netherlands Research Centre for Integrated Solid Earth Science (ISES). The reproducible numeric examples in this paper use the Madagascar open-source software package freely available from http://www.ahay.org and the one-way prestack depth migration code freely available from http://janth.home.xs4all.nl. 
Green's function retrieval and imaging with MDD
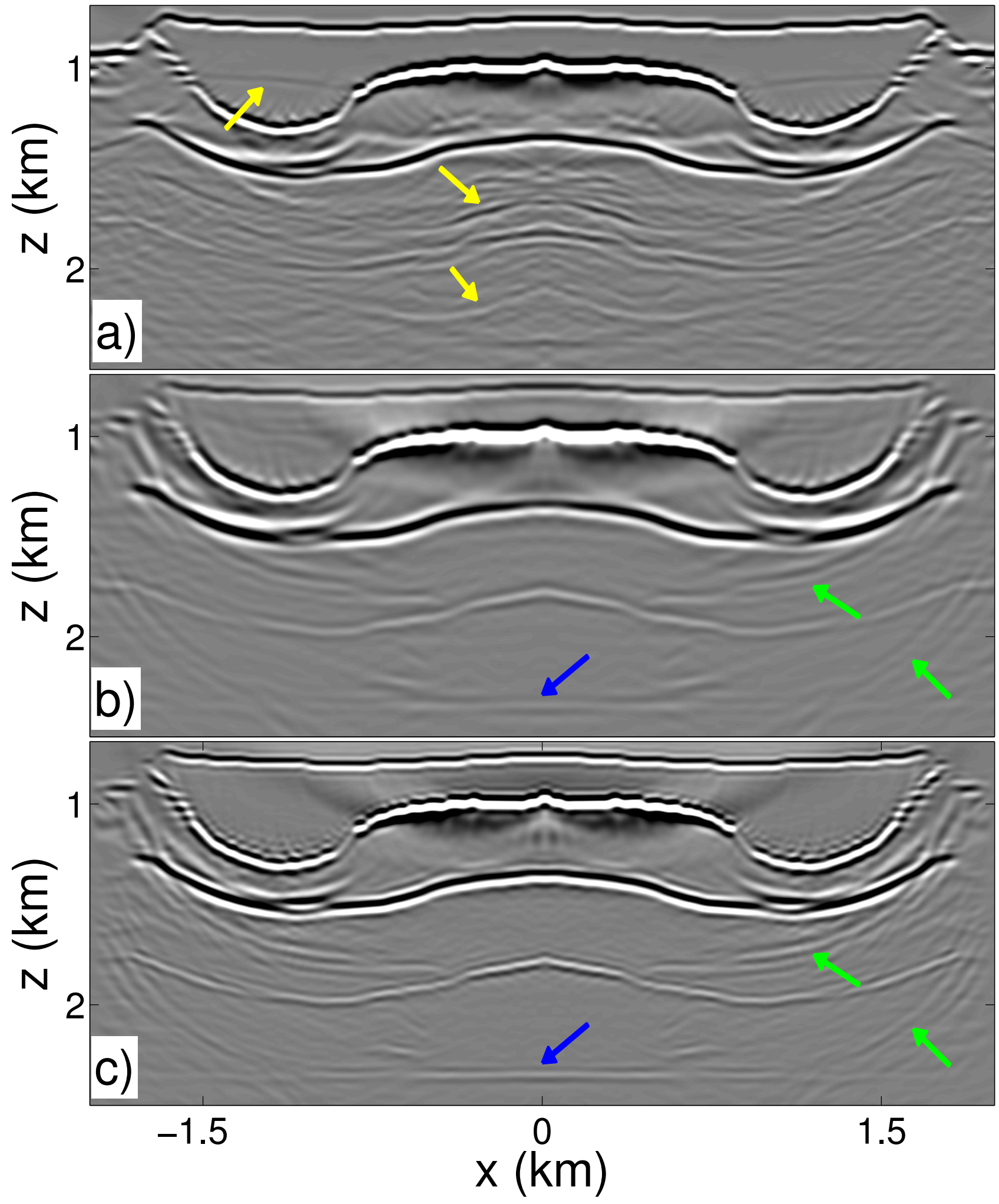

Figure 6: a) Image of the reflectors obtained with standard prestack migration. The yellow arrows indicate ghost images. b) Image of the reflectors obtained with the crosscorrelation function $C$. The green arrows indicate artifacts due to limited source aperture. The blue arrow indicates the flat reflector at $z=2.3 \mathrm{~km}$. c) Image of the reflectors obtained with multidimensional deconvolution. The green arrows indicate artifacts due to limited source aperture. The blue arrow indicates the flat reflector at $z=2.3 \mathrm{~km}$. 
http://dx.doi.org/10.1190/segam2013-0406.1

\section{EDITED REFERENCES}

Note: This reference list is a copy-edited version of the reference list submitted by the author. Reference lists for the 2013 SEG Technical Program Expanded Abstracts have been copy edited so that references provided with the online metadata for each paper will achieve a high degree of linking to cited sources that appear on the Web.

\section{REFERENCES}

Amundsen, L., 2001, Elimination of free-surface related multiples without need of the source wavelet: Geophysics, 66, 327-341, http://dx.doi.org/10.1190/1.1444912.

Artman, B., I. Podladtchikov, and B. Witten, 2010, Source location using time-reverse imaging: Geophysical Prospecting, 58, 861-873, http://dx.doi.org/10.1111/j.1365-2478.2010.00911.x.

Berkhout, A. J., 1997, Pushing the limits of seismic imaging, Part I: Prestack migration in terms of double dynamic focusing: Geophysics, 62, 937-953, http://dx.doi.org/10.1190/1.1444201.

Berkhout, A. J., and D. J. Verschuur, 1997, Estimation of multiple scattering by iterative inversion, Part I: Theoretical considerations: Geophysics, 62, 1586-1595, http://dx.doi.org/10.1190/1.1444261.

Claerbout, J. F., 1985, Imaging the earth's interior: Blackwell Scientific.

Etgen, J., and C. Regone, 1998, Strike shooting, dip shooting, widepatch shooting — Does prestack depth migration care? A model study: 68th Annual International Meeting, SEG, Expanded Abstracts, 6669.

Haffinger, P., and D. J. Verschuur, 2012, Estimation and application of near-surface full waveform redatuming operators: Geophysical Prospecting, 60, no. 2, 270-280, http://dx.doi.org/10.1111/j.1365-2478.2011.00983.x.

Jakubowicz, H., 1998, Wave equation prediction and removal of interbed multiples: 68th Annual International Meeting, SEG, Expanded Abstracts, 1527-1530.

Keho, T. H., and P. G. Kelamis, 2012, Focus on land seismic technology: The near-surface challenge: The Leading Edge, 31, 62-68, http://dx.doi.org/10.1190/1.3679329.

Sava, P., and B. Biondi, 2004, Wave-equation migration velocity analysis. II: Subsalt imaging examples: Geophysical Prospecting, 52, no. 6, 607-623, http://dx.doi.org/10.1111/j.1365-2478.2004.00448.x.

ten Kroode, F., 2002, Prediction of internal multiples: Wave Motion, 35, no. 4, 315-338, http://dx.doi.org/10.1016/S0165-2125(01)00109-3.

Thorbecke, J., 1997, Common focus point technology: Ph.D. dissertation, Delft University of Technology.

van der Neut, J., J. Thorbecke, K. Mehta, E. Slob, and K. Wapenaar, 2011, Controlled-source interferometric redatuming by crosscorrelation and multidimensional deconvolution in elastic media : Geophysics, 76, no. 4, SA63-SA76, http://dx.doi.org/10.1190/1.3580633.

van Groenestijn, G. J. A., and D. J. Verschuur, 2009, Estimation of primaries and near-offset reconstruction by sparse inversion: Marine data applications: Geophysics, 74, no. 6, R119-R128, http://dx.doi.org/10.1190/1.3213532.

Verschuur, D. J., A. J. Berkhout, and C. P. A. Wapenaar, 1992, Adaptive surface-related multiple elimination: Geophysics, 57, 1166-1177, http://dx.doi.org/10.1190/1.1443330. 
Wapenaar, K., F. Broggini, E. Slob, and R. Snieder, 2013, Three-dimensional single-sided Marchenko inverse scattering, data-driven focusing, Green's function retrieval, and their mutual relations:

Physical Review Letters, 110, no. 8, 084301, http://dx.doi.org/10.1103/PhysRevLett.110.084301.

Wapenaar, K., F. Broggini, and R. Snieder, 2012, Creating a virtual source inside a medium from reflection data: Heuristic derivation and stationary-phase analysis: Geophysical Journal International, 190, 1020-1024, http://dx.doi.org/10.1111/j.1365-246X.2012.05551.x.

Wapenaar, K., J. van der Neut, E. Ruigrok, D. Draganov, J. Hunziker, E. Slob, J. Thorbecke, and R. Snieder, 2011, Seismic interferometry by crosscorrelation and by multidimensional deconvolution: A systematic comparison: Geophysical Journal International 185, 1335-1364, http://dx.doi.org/10.1111/j.1365-246X.2011.05007.X.

Weglein, A., F. Gasparotto, P. Carvalho, and R. Stolt, 1997, An inverse-scattering series method for attenuating multiples in seismic reflection data: Geophysics, 62, 1975-1989, http://dx.doi.org/10.1190/1.1444298. 